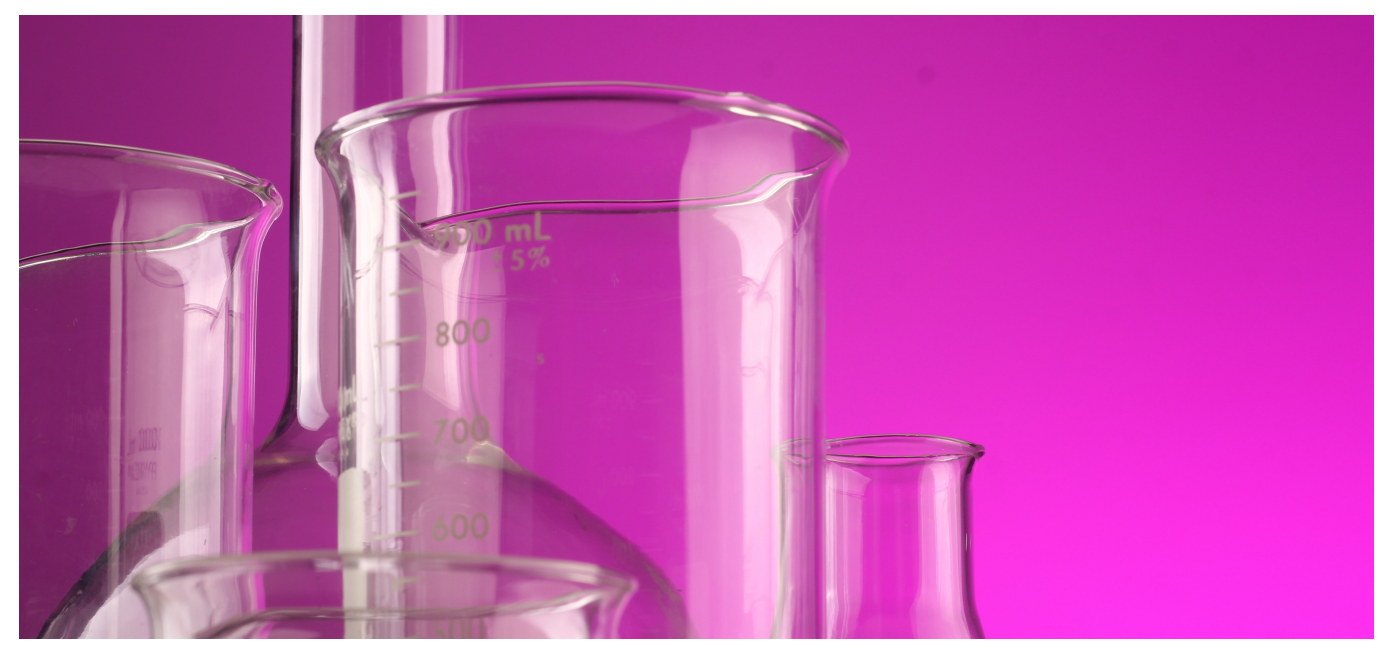

\title{
Systematic Review of the Use of Biomarkers in Early Breast Cancer Diagnosis
}

Ana Pedro ${ }^{1}$, rod tucker ${ }^{2}$

1 University of Hull

2 The Robert Gordon University

\section{Abstract}

Early detection of breast cancer improves prognosis. Unfortunately, in many instances patients' tumours are diagnosed following metastasis, thus making treatment more difficult and worsening their prognosis. The development of a specific biomarker for the early detection of breast cancer could potentially allow more women to be successfully treated yet at present there no validated biomarkers available. Only a few biomarkers (such as HER-2/neu, estrogen receptor, and progesterone receptor) have a limited utility for diagnosis and prognosis. Thus, there is a great need for new biomarkers for breast cancer. Here we review the current state of the art relative to the use of biomarkers in early breast cancer screening and diagnosis.

\section{Definitions}


True positive fraction

Defined by Marg aret Pepe

True negative fraction

Defined by Margaret Pepe

\section{Introduction}

Worldwide breast cancer $(\mathrm{BC})$ affects around 8 million women with the most common form, accounting for $70 \%$, being estrogen-receptor positive $(E R+)^{[1][2]}$.

Currently, women with suspected breast cancer are referred for mammography screening (MS) and a subsequent biopsy for confirmation of the diagnosis. However, MS is not diagnostic and can result in up 80\% false positives so that only around one in 4 women with an abnormal result will be found to have cancer after further inspection of abnormal areas with diagnostic mammography (DM) and eventually a biopsy ${ }^{[3][4]}$. Early detection of $\mathrm{BC}$ is associated with a better prognosis ${ }^{[5]}$, with the 5 -year survival for stage $1 \mathrm{BC}$ being $90 \%$ but only $13 \%$ for stage $4^{[2]}$. However, in up to $5 \%$ of patients, when the disease is detected the cance has metastasised, which is associated with a worse $\operatorname{prognosis}^{[6]}$.

The use of disease specific specific biomarkers offers the potential of earlier detection of BC and a timelier access to treatment which would improve prognosis, however, to date, no such validated biomarkers exist. Although current markers (such as HER2/neu, estrogen receptor (ER) and progesteron receptor (PR)) are useful prognostic tools that assist with disease management, they are not sufficiently discriminatory as although these markers are significantly raised in BC are also present in normal breast cells. Thus, there is a need for biomarkers which are only present in patients with $\mathrm{BC}^{[7]}$ and not in healthy women or women suffering from other pathologies such as ovarian cancer ${ }^{[8]}$, endometrial carcinoma ${ }^{[9]}$, endometriosis ${ }^{[10]}$, fibroids $^{[11][12]}$, cardiovascular disease ${ }^{[13]}$, Alzheimer's disease ${ }^{[14]}$ or colorectal cancer ${ }^{[15]}$ where these markers maybe raised as well. Also, these markers may detect ER+ or HER2 + BC, but they can't neither detect triple-negative BC or disting uish between lymph node negative (LN-) or lymph node positive $(\mathrm{LN}+)$ or metastatic $B C$.

A growing number of early diagnostic biomarkers are currently under investigation whereas others are targeted at prediction of metastatic behaviour and selection of therapy ${ }^{[16]}$, though at present none of these are used in clinical practice.

Here, we review the current data on biomarkers intended for the early detection of BC.

\section{Methods}


Searches were conducted using Pubmed, Scopus, CAB abstracts, Cochrane Library, Web of science core collection, NCRI cancer research database, Google Scholars, Embase and CINAHL, for articles published in the last 5 years.
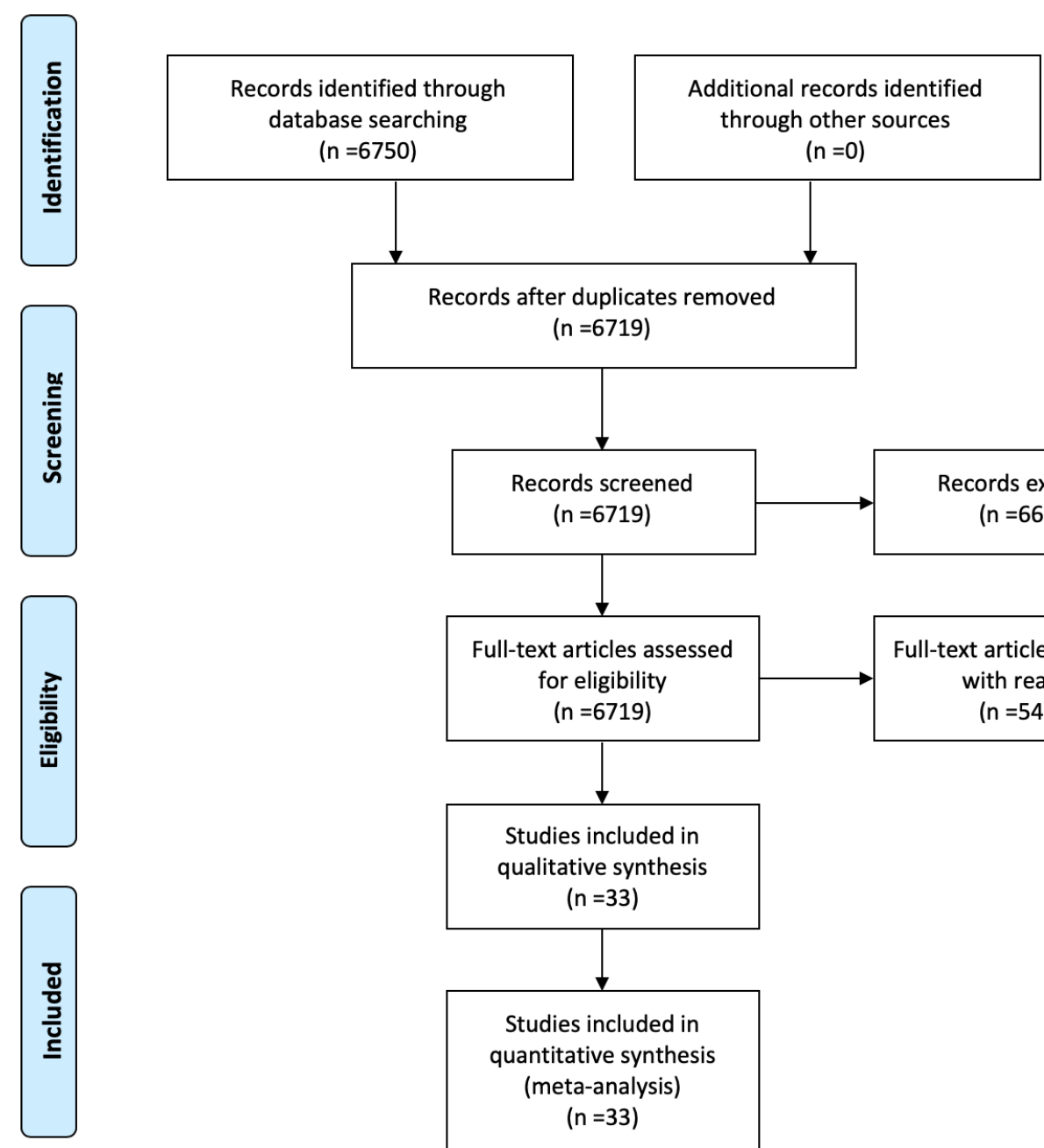

$(n=6719)$

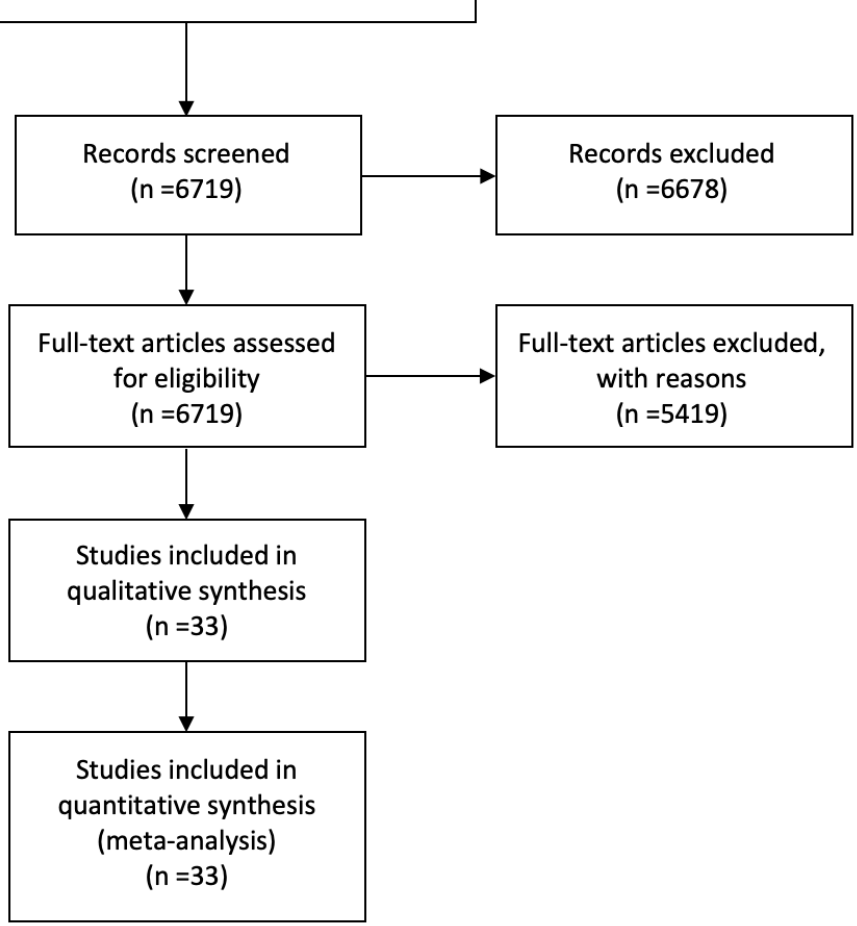

PRISMA 2009 Flow Diagram

\section{Results and Discussion}

As shown in the Prisma flow diagram, the search using the different databases and the criteria mentioned above yielded the articles mentioned in Table 1. Most of the developed biomarkers are proteins, RNA or DNA and a small proportion are based on tumor mechanical properties. Most came from studies in plasma/serum/blood and a minority from tissues, nipple fluid, urine or saliva. The biomarker which is closest to clinical use is the one described by Shimomura et al, $2016^{[17]}$ but is not yet validated and is based on miRNA detection what makes difficult to develop a simple lateral flow assay because miRNA needs to be isolated, amplified and labelled prior to testing it in the lateral flow assay strip ${ }^{[18][19]}$. 
Also, the use of protein panels that detect multiple biomarkers may enhance sensitivity and specificity in a clinical setting [20], however none of the multiple biomarkers shown in Table 1 was also yet validated.

In addition, most of the tests do not disting uish between LN- or LN- cancers or have been validated by the inclusion of advanced BC samples for comparison or specify the BC subtypes analysed what does not make possible to disting uish those cancers that are prone to progress and need treatment.

The ideal test would be specific for biomarkers that could be incorporated in a lateral flow assay and be developed to test a single drop of blood or other fluid directly into a testing strip, offering a fast and accurate diagnosis to allow a quick and adequate therapeutic intervention ${ }^{[21]}$. These tests would allow the patient to be selected for auxiliary imaging based on the presence of specific biomarkers which would specifically indicate the presence of occult cancer in patients with a normal SM.

\section{Data and software availability}

All data underlying the results is available as part of the article.

\section{Competing interests}

Biomarkers, Ltd, a split - off Roma Laboratories, Ltd is a company dedicated to the development of biomarkers for cancer.

\section{Grant information}

The authors declare no grants were involved in supporting this work.

\section{Acknowledments}

To University of Hull, University of York and Robert Gordon University libraries for use of their resources

\section{Supplementary Material}




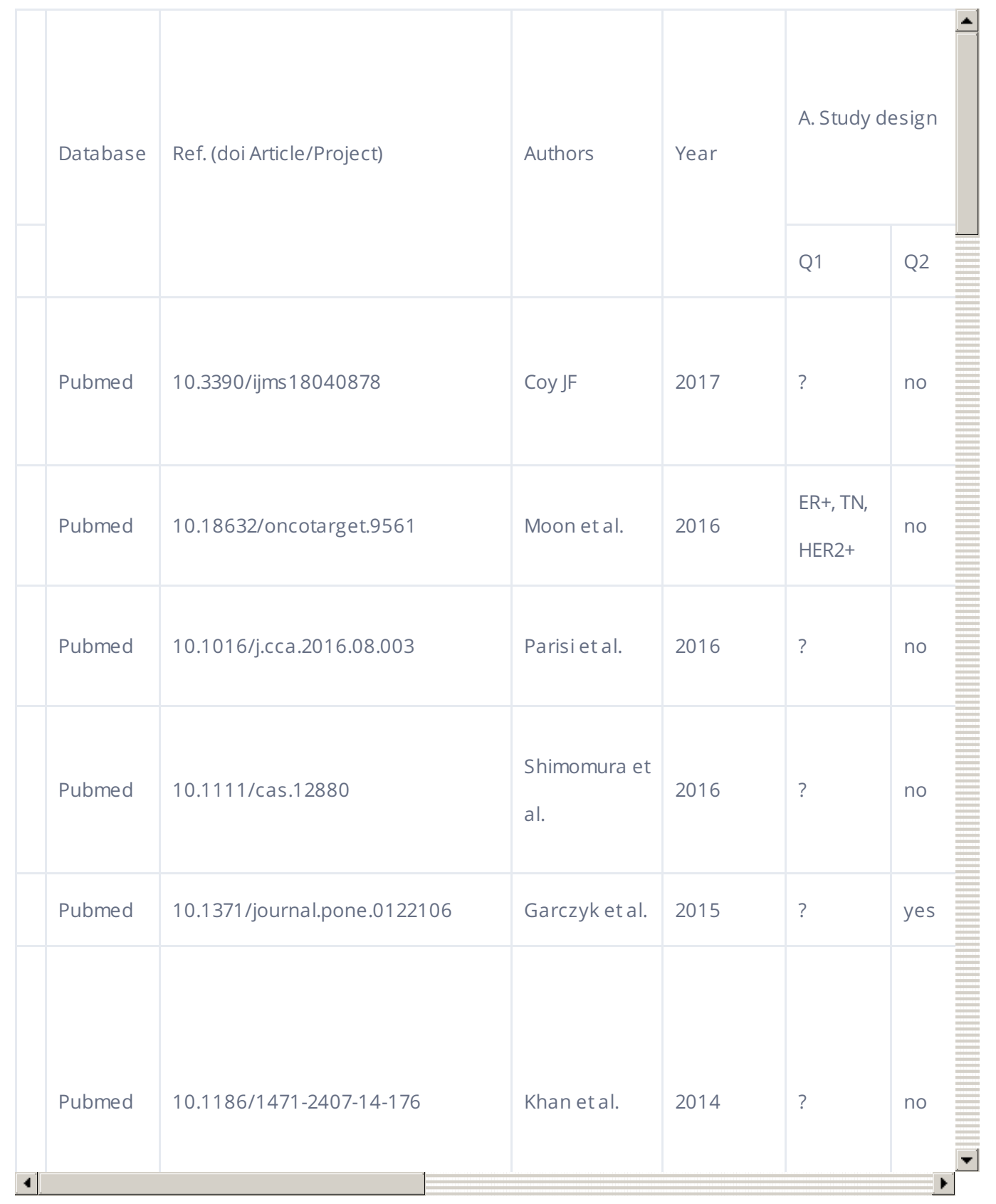

Table 1 
Supplementary Material

SM1: PRISMA flow diagram

SM2: STANDARD TOOL

A- Study design

1. Breast cancer subtypes analysed?

2. Distinguishes lymph node (LN) negative (-) and LN positive (+) breast cancer or precancerous lesions?

3. Control samples include healthy volunteers and patients with other conditions rather than breast cancer and advanced breast cancer patients?

\section{References}

1. ^ https://www.macmillan.org.uk/information-and-support/breast-

cancer/treating/treatment-decisions/understanding-your-diagnosis/receptors-forbreast-cancer.html

2. ${ }^{a}, \mathrm{~b}$ https://www.wcrf.org/int/cancer-facts-figures/data-specific-cancers/breastcancer-statistics

3. ^ 4) Ekpo EU, Alakhras M, Brennan P. (2018). Errors in Mammography Cannot be Solved Through Technology Alone. Asian Pac J Cancer Prev., vol. 19(2):291-301 
4. ^ https://www.nhs.uk/conditions/breast-cancer-screening/results/

5. ^ https://www.cancerresearchuk.org/about-cancer/cancer-symptoms/why-is-earlydiagnosis-important

6. ${ }^{\wedge}$ https://www.cancerresearchuk.org/health-professional/cancer-statistics/statisticsby-cancer-type/breast-cancer/survival\#By

7. ^ D. Mouttet, M. Laé, M. Caly, D. Gentien, S. Carpentier, H. Peyro-Saint-Paul. (2016). Estrogen-Receptor, Progesterone-Receptor and HER2 Status Determination in Invasive Breast Cancer. Concordance between Immuno-Histochemistry and

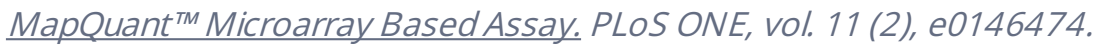

8. ^Ilaria De Stefano, Gian Franco Zannoni, Maria Grazia Prisco, Anna Fagotti, Lucia Tortorella, Giuseppe Vizzielli. (2011). Cytoplasmic expression of estrogen receptor beta (ERß) predicts poor clinical outcome in advanced serous ovarian cancer. Gynecologic Oncology, vol. 122 (3), 573-579.

9. ^ A Lebeau, TJ Grob, FHolst, N Seyedi-Fazlollahi, H Moch, Luigi Terracciano. (2008). Oestrogen receptor gene (ESR1) amplification is frequent in endometrial carcinoma and its precursor lesions. J. Pathol., vol. 216 (2), 151-157.

10. ^ Paola Montagna, Silvia Capellino, Barbara Villaggio, Valentino Remorgida, Nicola Ragni, Maurizio Cutolo. (2008). Peritoneal fluid macrophages in endometriosis: correlation between the expression of estrogen receptors and inflammation. Fertility and Sterility, vol. 90 (1), 156-164.

11. ^ Pamela L. Strissel, Justine Swiatek, Peter Oppelt, Stefan P. Renner, Matthias W. Beckmann, Reiner Strick. (2007). Transcriptional analysis of steroid hormone receptors in smooth muscle uterine leiomyoma tumors of postmenopausal patients. The Journal of Steroid Biochemistry and Molecular Biology, vol. 107 (1-2), 42-47.

12. ^ Panagiotis Bakas, Angelos Liapis, Spiros Vlahopoulos, Maria Giner, Stella Logotheti, Georgios Creatsas. (2008). Estrogen receptor $\alpha$ and $\beta$ in uterine fibroids: a basis for altered estrogen responsiveness. Fertility and Sterility, vol. 90 (5), 1878-1885.

13. ^Valentin Leibetseder, Susanne Humpeler, Andreas Zuckermann, Martin Svoboda, Theresia Thalhammer, Wolfgang Marktl. (2010). Time dependence of estrogen receptor expression in human hearts. Biomedicine \& Pharmacotherapy, vol. 64 (3), 154-159.

14. ^ Chunyu Wang, Fan Zhang, Siruiliang, Sandra L. Siedlak, Lu Shen, George Perry. (2016). Estrogen receptor- $\alpha$ is localized to neurofibrillary tangles in Alzheimer's disease. Sci Rep, vol. 6 (1)

15. ` Jeffrey S. Ross, Marwan Fakih, Siraj M. Ali, Julia A. Elvin, Alexa B. Schrock, James Suh. 
(2018). Targeting HER2 in colorectal cancer: The landscape of amplification and short variant mutations in ERBB2 and ERBB3. Cancer, vol. 124 (7), 1358-1373.

16. ^ https://www.cancer.net/research-and-advocacy/asco-care-and-treatmentrecommendations-patients/biomarkers-guide-treatment-early-stage-breast-cancer

17. ^ Akihiko Shimomura, Sho Shiino, Junpei Kawauchi, Satoko Takizawa, Hiromi Sakamoto, Juntaro Matsuzaki. (2016). Novel combination of serum microRNA for detecting breast cancer in the early stage. Cancer Sci, vol. 107 (3), 326-334.

18. ^Shao-YiHou, Yi-Ling Hsiao, Ming-Shu Lin, Chun-Che Yen, Chi-Sheng Chang. (2012). MicroRNA detection using lateral flow nucleic acid strips with gold nanoparticles. Talanta, vol. 99, 375-379.

19. ^ Na Ying, Chuanjing Ju, Xiuwei Sun, Letian Li, Hongbiao Chang, Guangping Song. (2017). Lateral flow nucleic acid biosensor for sensitive detection of microRNAs based on the dual amplification strategy of duplex-specific nuclease and hybridization chain reaction. PLOS ONE, vol. 12 (9), e0185091.

20. `Alan B Hollingsworth, David E Reese, Medical Director, Mercy Women's Center, Mercy Hospital-OKC, Oklahoma City, OK, US, Chief Scientific Officer and Chief Executive Officer, Provista Diagnostics, Inc., New York, NY, US. (2014). Potential Use of Biomarkers to Augment Clinical Decisions for the Early Detection of Breast Cancer.

21. ^Joshua Campbell, Joseph Balhoff, Grant Landwehr, Sharif Rahman, Manibarathi Vaithiyanathan, Adam Melvin. (2018). Microfluidic and Paper-Based Devices for Disease Detection and Diagnostic Research. IJMS, vol. 19 (9), 2731. 